\title{
Comparative Analysis of various Illumination Normalization Techniques for Face Recognition
}

\author{
Tripti Goel \\ GPMCE, Delhi
}

\author{
Vijay Nehra \\ BPSMV, Khanpur
}

\author{
Virendra P.Vishwakarma \\ JIIT, Noida
}

\begin{abstract}
The change in facial appearance due to illumination variation degrades face recognition systems performance considerably. In this paper, various states of art illumination normalization techniques have been explained and compared. The classification of the image recognition has been done using artificial neural networks (ANN). We have compared four illumination normalization methods which are (1) discrete cosine transform (DCT) with rescaling of low frequency coefficients (2) discrete cosine transform (DCT) with discarding of low frequency coefficients (3) homomorphic filtering (HF) (4) gamma intensity correction (GIC). These methods are evaluated and compared on Yale and Yale B Faces databases.
\end{abstract}

Keywords: Discrete cosine transform (DCT), homomorphic filtering (HF), gamma intensity correction (GIC), artificial neural networks (ANN)

\section{INTRODUCTION}

During past several years, face recognition have received significant attention to establish personal authenticity. Several vendors around the world claim the working of recognition system successfully. It has been proved by the face recognition conferences such as AFGR[1] and ABVP[2]. Main applications of face recognition system are in public security, law enforcement , and commerce such as mug shot database matching, identity authentication for credit card or driver license, access control, information security, and intelligent surveillance[3]. So far, face recognition technology have received very high accuracy under restricted environment such as frontal faces with indoor lighting conditions. However, illumination variation is still a challenging problem in this area. The same person can appear greatly different under varying lighting conditions [4-7]. Therefore, performance of automatic face recognition deteriorates.

Many approaches have been proposed to recognize the human faces under different lighting conditions. Model based approaches attempt to model the variations caused by variation in illumination.[8]. On the other hand, preprocessing based approaches transforms image directly without any prior knowledge or assumption of illumination [9]. However, model based approaches require the model of lighting which is difficult to get for real applications. So preprocessing -based approaches are widely used in which the illumination effect is removed in the preprocessing stage before classification.
In the early face recognition approaches, principal component analysis (PCA) was applied at the recognition stage [10-13]. Advances in this algorithm were linear discriminant analysis (LDA), local binary patterns (LBP) [15], elastic bunch graph matching (EBGM) and independent component analysis (ICA) [14]. These algorithms demonstrated a good performance in various face recognition problems. However, if the lighting conditions varied, the recognition performance appears unstable. In this paper, artificial neural network (ANN) is used for training and testing of face recognition problem. An ANN is developed with a systematic step by step procedure which optimizes the learning capability. The input/output training data is fundamental for these networks as it conveys the information necessary for the optimal operating point.

Before applying ANN, we have to normalize the variation in the appearance by the illumination compensation and normalization methods. Main methods used in this paper are: (1) DCT with rescaling of low frequency coefficients is employed to utilize the fact that low frequency DCT coefficients are correlated with illumination variations. (2) DCT with discarding low frequency coefficients, in which an appropriate number of low frequency DCT coefficients are truncated to zero. (3) Homomorphic Filtering (HF) to reduce illumination effect while the reflectance after this procedure will be closer to the original reflectance. (4) Gamma Intensity Correction (GIC) method to normalize the overall image intensity to a given intensity level.

The rest of the paper is organized as follows: In section 2, the detailed theoretical background of the illumination normalization methods are given. In section 3, we trained ANN using the given data after using the normalization techniques. The results of all the methods used for illumination normalization are also given here. In section 4 , we concluded the paper and lists possible improvements and further work.

\section{INTENSITY TRANSFORMATION FUNCTION FOR ILLUMINATION NORMALIZATION}

In intensity transformation process, intensity transformation operates directly on the pixels of an image. The intensity transformation process is denoted by the expression:

$$
g(x, y)=T[f(x, y)]
$$

where $f(x, y)$ is the input image and $g(x, y)$ is the output image after illumination normalization [16]. Illumin ation normalization method attempts to obtain a face image which is the image of 
the same face captured under the predefined known lighting conditions. This normalization technique enhances the contrast of the image to increase the recognition rate for face recognition.

\subsection{DCT with rescaling coefficient}

First step in face recognition by rescaling of DCT coefficients, is histogram equalization. Histogram equalization increases the local contrast of object in the image. But, by using histogram equalization, illumination variations are not affected. Only illumination variations are shifted to the upward in the gray scale. This problem can be solved by our approach DCT with rescaling coefficients. It is because low frequency DCT coefficients are correlated with illumination variations. Elaboration of these steps have been explained below

\subsubsection{Histogram Equalization (HE)}

Histogram Equalization is the most common histogram normalization or gray level transform. It produces an image with equally distributed brightness levels over the whole brightness scale. It modifies the dynamic range of the image and hence normalizes the illumination of the image [16]. This can be explained as below: gray level is

The probability density function of the transformed

$p(s)=\left[p_{r}\left(r \frac{d r}{d s}\right)\right]$

Consider the transformation function

$$
s=T(r)=\int_{0}^{r} p(w) d w
$$

Where $w$ is a dummy variable of integration. The rightmost side of equation (3) is cumulative distribution function (CDF) of $r$. Take derivative of $s$ with respect to $r$ is

$$
\frac{d s}{d r}=p_{r}(r)
$$

Substituting dr/ds into equation (2) y ields

$$
p_{r}(s)=\left[p_{r}(r) \frac{1}{p_{r}(r)}\right]
$$

This is a uniform density in the interval of the transform variable $\mathrm{s}$. This result indicates that using a transformation function equal to the cumulative distribution of $\mathrm{r}$ produces an image whose gray level have a uniform density. This result can have a considerable effect in the appearance of a $\mathrm{n}$ image.

\subsubsection{Discrete Cosine Transform (DCT)}

The discrete cosine transform (DCT) represents an image as a sum of sinusoids of vary ing magnitudes and frequencies [17]. It is a popular technique for image compression. Example for DCT is in JPEG image compression. The forward 2D DCT of a MXN size image is defined as:

$$
\begin{aligned}
& B_{p q}=\alpha_{p} \alpha_{q} \sum_{m=0}^{M-1} \sum_{n=0}^{N-1} A_{m n} \cos \frac{\pi(2 m+1) p}{2 M} \cos \frac{\pi(2 n+1) q}{2 N}, \begin{array}{l}
0 \leq p \leq M-1 \\
0 \leq q \leq N-1
\end{array} \\
& \alpha_{p}=\left\{\begin{array}{ll}
1 / \sqrt{M}, & p=0 \\
\sqrt{2 / M}, & 1 \leq p \leq M-1
\end{array} \alpha_{q}= \begin{cases}1 / \sqrt{N}, & q=0 \\
\sqrt{2 / N}, & 1 \leq q \leq N-1\end{cases} \right.
\end{aligned}
$$

The inverse of DCT is defined as:

$$
A_{m n}=\sum_{p=0}^{M-1} \sum_{q=0}^{N-1} \alpha_{p} \alpha_{q} B_{p q} \cos \frac{\pi(2 m+1) p}{2 M} \cos \frac{\pi(2 n+1) q}{2 N}, \begin{aligned}
& 0 \leq m \leq M-1 \\
& 0 \leq n \leq N-1
\end{aligned}
$$

Where, $\mathrm{m}$ and $\mathrm{n}$ are the spatial coordinates in the image block and $\mathrm{p}$ and $\mathrm{q}$ are coordinates in the DCT coefficients block. $\alpha_{p} \alpha_{q}$ are the basis functions of DCT.

Figure 1 shows the properties of the DCT coefficients in $M \times N$ blocks with the zigzag pattern used by JPEG compression to process the DCT coefficients. The DC coefficient at the upper left corner of the image block holds most of the image energy and represents the proportional average of $M \times N$ blocks. The remaining coefficients denote the intensity changes among the block images and referred to as AC coefficients.

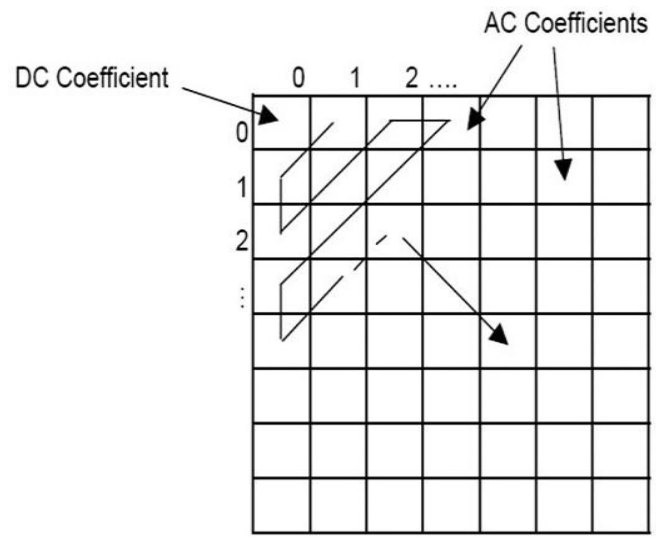

Figure 1. B lock feature of DCT coefficients and their selection in zigzag pattern.

\subsubsection{DCT with rescaling coefficients}

In a face image, low frequency DCT coefficients are correlated with illumination variations. Therefore, illumination variation will lie in the low frequency components. We can select low frequency components for rescaling [18]. So, illumination variations under different lighting conditions can be significantly reduced by downscaling low frequency coefficient. The value by which the value of low frequency components are rescaled down are called Rescaling Down Factor (RDF). The number of low frequency DCT coefficient are to be rescaled is 
denoted by $C_{\text {resc. }}$. The actual number of low frequency DCT coefficients, selected for rescaling, can be calculated using following equation:

Total number of low frequency DCT coefficients $=C_{\text {resc }} . X\left(C_{\text {resc }}\right.$ $-1)-1$

\subsection{DCT with discarding low coefficients}

In this technique, illumination variation has been compensated by truncating low frequency components [19]. It is because illumination variation lies in the low frequency band. Evidently, the resulting results will act as an ideal high pass filter.

\subsection{Homomorphic Filtering $(H F)$}

An image $I(x, y)$ may be characterized by two components: (1) the amount of source light incident on the scene being viewed and (2) the amount of light reflected by the object in the scene. These components are called illumination and reflectance components. So, an image can be represented as illuminationreflectance model and expressed as:

$$
I(x, y)=L(x, y) R(x, y)
$$

Where, $L(x, y)$ is the illumination component and $R(x, y)$ is the reflectance component.

This illumination-reflectance model can be used as the basis for a frequency domain procedure for improving the appearance of an image by simultaneous brightness range compression and contrast enhancement. Homomorphic filtering is a frequency domain filtering process that does that. After Homomorphic filtering [20] process, $I(x, y)$, the process illumination normalization should drastically reduced due to high pass filtering effect, while reflectance $R(x, y)$ after this procedure should still be very close to original reflectance. The steps for Homomorphic filtering are as follows:

Step 1: First we have to take logarithm to transfer multiplication to addition, so that we could use multiplication or convolution property of Fourier Transform

$$
\begin{aligned}
& Z(x, y)=\ln [I(x, y)]=\ln [R(x, y) \cdot L(x, y)]= \\
& \ln [R(x, y)]+\ln [L(x, y)]
\end{aligned}
$$

Step 2 : Use 2D Fourier Transform on equation (10)

Or,

$$
\begin{aligned}
& F\{Z(x, y)\}=F\{\ln [R(x, y)]\}+F\{\ln [l(x, y)]\} \\
& Z(u, v)=F_{R}(u, v)+F_{L}(u, v)
\end{aligned}
$$

Step3 : Suppress low frequency component in frequency domain by using homomorphic filtering process

$$
\begin{array}{r}
\mathrm{S}(u, v)=H(u, v) \cdot Z(u, v)= \\
H(u, v) \cdot F_{R}(u, v)+H(u, v) \cdot F_{L}(u, v)
\end{array}
$$

Where, $H(u, v)$ is the homomorphic filtering function.

Step 4: Take inverse transform of equation (13)

$$
\begin{gathered}
s(x, y)=F^{-1}\{S(u, v)\}= \\
F^{-1}\left\{H(u, v) \cdot F_{R}(u, v)\right\}+F^{-1}\left\{H(u, v) \cdot F_{L}(u, v\}\right)
\end{gathered}
$$

Step 5: Take exponential operation to get the desired enhanced image

$$
I^{\prime}(x, y)=e^{s(x, y)}
$$

By taking exponential, the desired filtered and enhanced image I'(x,y)can be obtained. The complete procedure for homomorphic filtering is summarized in fig2 below:

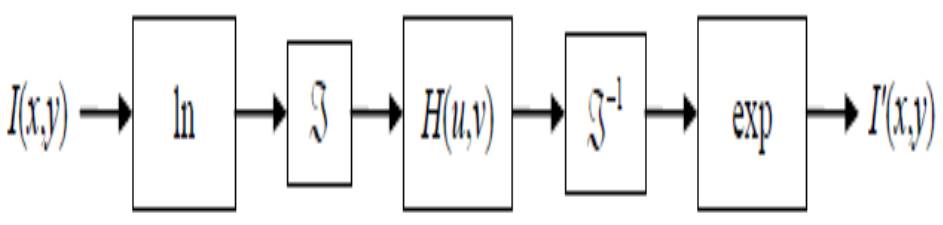

Figure 2 Homomorphic Filtering process

\subsection{Gamma Intensity Correction (GIC)}

Gamma correction has become increasingly important in the past few years, as use of the digital images for commercial purpose has increased gamma correction produces an output that is close in appearance to the original image. Gamma correction method corrects the overall brightness of the image to a predefined canonical face images. It is formulated as follows:

Predefine a canonical image $I_{0}$, which has been lighted under some normal lighting condition. The given face $I$, captured under some unknown lighting condition. Its canonical image is computed by a Gamma transform pixel by pixel over the image position $x, y$ :

$$
I_{x y}^{\prime}=G\left(I_{x y} ; \gamma^{*}\right)
$$

where the Gamma coefficient $\gamma *$ is computed by the following optimization process, which aims at minimizing the difference between the transformed image and the predefined normal face image $I_{0}$

$$
\gamma^{*}=\arg \min _{\gamma} \sum_{x . v}\left[G\left(I_{x y} ; \gamma\right)-I_{0}(x, y)\right]^{2},
$$

where $I x y$ is the gray-level of the image position $x, y$; and

$$
G\left(I_{x y} ; \gamma\right)=c \cdot I_{x y}^{\frac{1}{\gamma}},
$$

is the Gamma transform; $c$ is a gray stretch parameter, and $\gamma$ is the Gamma coefficient.

Thus, from the above equations, gamma has the effect of adjusting overall brightness of all the face images to the same level as that of the common normal face $I O$. 


\section{EXPERIMENTAL RESULTS 3.1 Face Databases Used}

In this paper, the experiments are carried out on the Yale Face Database B. This database contain face images with large illumination variations. Results are evaluated and compared for different illumination normalization methods discussed before.

Yale Face Database B: This database contains 10 subjects which contains 5760 single light source images under 576 different viewing conditions $(9$ poses $X 6$ illumination condition). Every subject of one individual is divided into five subset according to the angle between light source direction and camera axis[21]. The azimuth and elevation of light source direction for these subsets are given in Table 1. For performing experiments, we used subset 1 as the training set and rest of four as the test sets. In this paper, comparison of various illu mination techniques will be done on this database.

\begin{tabular}{|c||c|c|c|c|c|}
\hline Subset & 1 & 2 & 3 & 4 & 5 \\
\hline Azimuth \& Elevation $\left({ }^{0}\right)$ & $0 \sim 12$ & $13 \sim 25$ & $26 \sim 50$ & $51 \sim 77$ & $>77$ \\
\hline Number of subset images & 60 & 120 & 120 & 140 & 190 \\
\hline
\end{tabular}

\section{Table 1. Subsets of Yale Face Database B.}

\subsection{Artificial Neural Networks}

Artificial Neural Networks are composed of simple elements operating in parallel. These elements are inspired by biological nervous system. Commonly, neural networks are adjusted, or trained, so that a particular input leads to a specific target output. In this paper we use Back Propagation Algorithm to solve the recognition problem. In this algorithm, input vectors and the corresponding target vectors are used to train a network until it can approximate a function, associate input vectors with a specific output vectors, or classify that vectors in an approximate way [22].

The architecture of network used for back propagation algorithm has been given in fig3. An elementary neuron with $\mathrm{R}$ inputs has been shown. Each input is weighted with an appropriate value w.

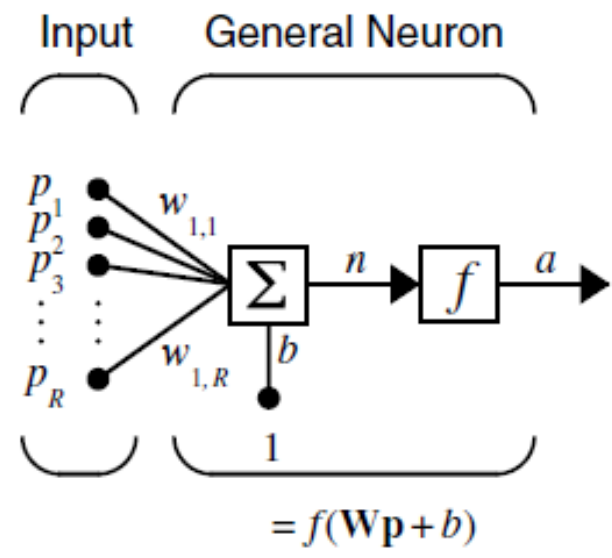

Fig 3. Neuron Model for Back Propagation Algorithm
In Back Propagation, standard Gradient Descent Algorithm is used, in which the network weights are moved along the negative of the gradient of the performance function. One iteration of algorithm can be written as;

$$
\mathbf{x}_{k+1}=\mathbf{x}_{k}-\alpha_{k} \mathbf{g}_{k}
$$

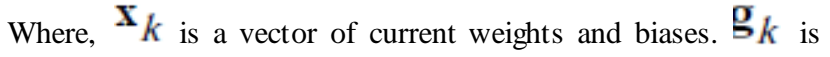
current gradient and $\alpha_{k}$ is the learning rate.

There are two different ways in which this gradient descent algorithm can be implemented: incremental mode and batch mode. In the incremental mode, the gradient is computed and the weights are updated after each input is applied to the network. In the batch mode all of the inputs are applied to the network before the weights are updated.

There are generally six steps in the training process:

(1) Assembling the training data

(2) Illumination normalization

(3) Create the new object

(4) Train the network

(5) Simulate the network response to the network inputs

(6) Calculation of recognition rate

This algorithm has been applied to all the techniques of illumination normalization discussed before. A comparison between all these techniques using Back Propagation algorithm has been given in next section.

\subsection{Method Tested}

(a) No Enhancement: In this method, we have not use any normalization technique. No filtering or equalization method. Artificial Neural Network has been trained according to the given data set as input to the network without any enhancement.(fig 4a)

(b) Histogram Equalization: In this method, the images are normalizes using Histogram Equalization method. Histogram Equalization enhances the contrast of images by transforming the values in an intensity image, so that the histogram of the output image has been uniformly distributed. (fig 4b).

(c) Discrete Cosine Transform (DCT) with rescaling coefficients: It is a popular image compression technique used in JPEG image compression. In this technique, illumination variation can be compensated by down scaling the low frequency DCT coefficients. (Fig 4c)

(d) Discrete Cosine Transform (DCT) discarding coefficients: In this technique, low frequency component can be removed by setting the low frequency DCT coefficient to zero. Setting the DCT coefficients to zero is equivalent to subtracting the product of the DCT basis image and the corresponding coefficient in th original image. 
(e) . Gamma Intensity Correction(GIC): In this method, Gamma function adjust the image intensity values

GIC method corrects the overall brightness of the image to a predefined canonical face image (fig $4 \mathrm{e}$ ). gamma correct ion can control the overall brightness of an image by changing the gamma parameter.

(f) Homomorphic Filtering $(H F)$ : In Homomorphic Filtering, the images are normalized using High Pass Filtering process in frequency domain. This high pass filtering is done by Homomorphic Filtering. After Homomorphic
Filtering (HF), illumination reduced drastically due high pass filtering effect, while reflectance is still very close to original reflectance. (fig $4 \mathrm{f}$ )

The face images after described normalization techniques is shown in fig 4..After doing normalization, back propagation method has been applied on Yale face database B.

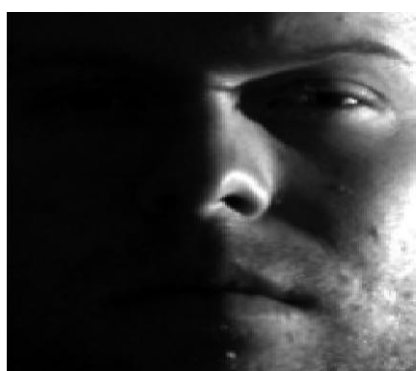

(a)

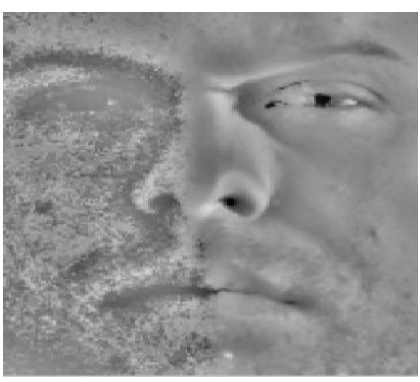

(d)

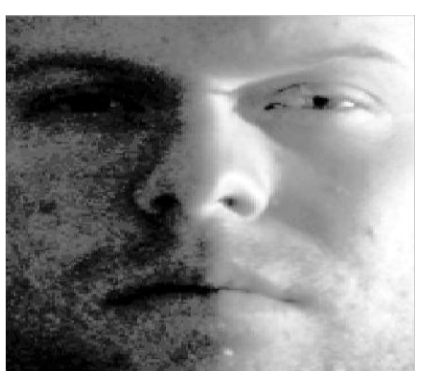

(b)

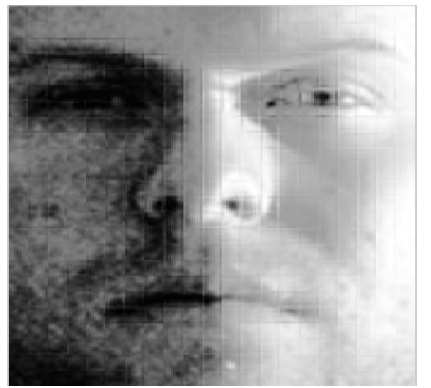

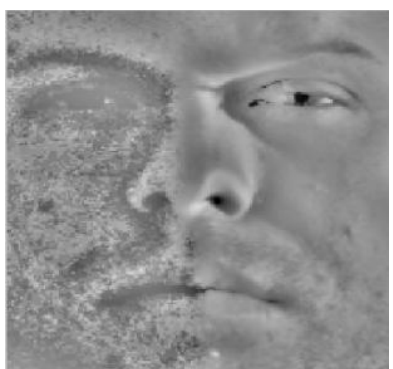

(c)

(e)

(f)

Fig 4 Example of images of Yale Face Database after applying illumination normalization techniques

\section{3-4 Experimental Results}

Experiments are conducted on the Yale B face database with above mentioned illumination normalization techniques. Yale face database is publicly available for studying pose and illumination problem. This database contains images of ten persons with 64 different illumination conditions. The images of every subject have been divided into five subsets according to the angle that light source direction makes with the camera axis. In this database, subset 1 will be used as the training and rest of four subsets will be used as the test set. First, the illumination variations are normalized by the normalization techniques described before. After that training and classification has been done by using back propagation algorithm. The comparison of different illumination normalization techniques has been given in table 2. The recognition error rate with subset 2 is zero or almost negligible. Since this set contains face images with small illumination variations, so in table2, the recognition error with subset 2 is not shown.
Table 2. Illumination rate comparisons of different illumination normalization methods on Yale Face Database B (subset 1 is used as the training images for each person)

\begin{tabular}{|c|c|c|c|}
\hline $\begin{array}{c}\text { Illumination } \\
\text { normalization } \\
\text { techniques }\end{array}$ & $\begin{array}{c}\text { Subset } \\
3\end{array}$ & $\begin{array}{c}\text { Subset } \\
4\end{array}$ & $\begin{array}{c}\text { Subset } \\
5\end{array}$ \\
\hline $\begin{array}{c}\text { No } \\
\text { Enhanceme nt }\end{array}$ & 3.3 & 47.142 & 85.789 \\
\hline $\begin{array}{c}\text { Histogram } \\
\text { Equalization }\end{array}$ & 0 & 31.42 & 43.157 \\
\hline
\end{tabular}




\begin{tabular}{|c|c|c|c|}
\hline $\begin{array}{c}\text { Gamma } \\
\text { Inte nsity } \\
\text { Correction }\end{array}$ & 0 & 27.12 & 36.482 \\
\hline $\begin{array}{c}\text { Homomorphic } \\
\text { Filte ring }\end{array}$ & 0 & 26.42 & 37.368 \\
\hline $\begin{array}{c}\text { DCT with } \\
\text { Dis carding } \\
\text { Coefficients }\end{array}$ & 1.2 & 25.743 & 17.222 \\
\hline $\begin{array}{c}\text { DCT with } \\
\text { Downscaling } \\
\text { Coefficients }\end{array}$ & 1.2 & 25.743 & 11.185 \\
\hline
\end{tabular}

4. Comparison

A comparison of different illumination normalization techniques has been given in table 2. These techniques have been trained using B ack Propagation Algorithm. As can be seen from table, DCT with downscaling coefficients gives the lowest error rate as compared to other techniques. Therefore, DCT with downscaling coefficients performs better and give better results than other techniques for illumination normalization.

\section{CONCLUSIONS}

In this paper, comparative analyses of various illumination normalization techniques have been presented. We aim to minimize the recognition error rate which occurs due to illumination variations. Training and recognition rate has been calculated using artificial neural networks. The comparison of method has been evaluated with the YaleB face database. The results show that proper rescaling down of sufficient number of low frequency DCT coefficients can give the better results for the face recognition. Further research can be conducted to improve recognition error rate more to deal with the problem of pose also.

\section{REFERENCES}

[1] Proceeding of the International Conferences on the Automatic Face and Gesture Recognition, 1995-1998

[2] Proceeding of the International Conferences on the Audio and Video Based Person Authentication, 199-1998.

[3] W. ZHAO, R. CHELLAPPA, P. J. PHILLIPS AND AROSENFELD, Face Recognition: A Literature Survey, ACM Computing Surveys 35 (4) (2003) pp. 399-458.

[4] R.CHELlAPPA, C. L. WILSON AND S. SIROHEY, Human and machine recognition of faces: a survey. Proceedings of IEEE 83 (5) (1995) pp. 705-740.

[5] P.N. BELHUMEUR AND D. J. KRIEGMAN, What is the set of images of an object under all possible illumination conditions. International Journal of Computer Vision 28 (3) (1998) pp. 245-260.
[6] S.Z LI AND A. K. JAIN, Handbook of Face Recognition, Springer, 2005.

[7] X. ZOU, J. KITTLER AND K. MESSER, Illumination Invariant Face Recognition: A Survey, in: Proceedings of IEEE International Conference on Biometric: Theory, Application and Systems (2007), pp. 1-8.

[8] P. N. Belhumeur, J. P. Hespanha, and D. J. Kriegman, "Eigenfaces vs. Fisherfaces: Recognition using class specific linear projection," IEEE Trans. on Patt. Ana. and Mach. Intell., vol. 19, no. 7, pp. 711-720, 1997.

[9] T. Cootes, G. Edwards, and C. Tay lor, "Active appearance models," IEEE Trans. on Patt. Ana. and Mach. Intell., vol. 23, no. 6, pp. 681-685, 2001.

[10] M. TURK AND A. PENTLAND, Eigenfaces for Recognition, J. Cognitive Neuroscience, 3 (1) (1991) pp. 71-96.

[11] Y. Adini, Y. Moses, and S. Ullman, "Face recognition: the problem of compensating for changes in illumination direction," IEEE Trans. PatternAnal. Mach. Intell., vol. 19, no. 7, pp. 721-732, Jul. 1997.

[12] K. DELAC, M. GRGIC AND S. GRGIC, Independent Comparative Study of PCA, ICA, and LDA on the FERET Data Set, International Journal of Imaging Systems and Technology, 15 (5) (2006) pp. 252-260

[13] S. Shan, W. Gao, B. Cao and D. Zhao, "Illumination Normalization for Robust Face Recognition Against Varying Lighting Conditions", Proc. IEEE Workshop on AMFG, pp. 157-164, 2003.

[14] Bruce A. Draper, Kyungim Baek, Marian Stewart Bartlett, "Recognizing faces with PCA and ICA", Computer vision and Image Understanding 91 (2003) 115-137

[15] T. Ahonen, A. Hadid, and M. Pietikainen, "Face Description with Local Binary Patterns: Application to Face Recognition”, IEEE Trans. Pattern Analysis and Machine Intelligence, vol. 28, no. 12, pp. 2037-2041, Dec 2006

[16] R.C. Gonzalez, R.E. Woods, "Digital Image Processing",Prentice Hall, Upper Saddle River, NJ, 2002

[17] Z. M. Hafed and M. D. Levine, "Face recognition using the discrete cosine transform," Int. J. Comput. Vis., vol. 43, no. 3, pp. 167-188, 2001.

[18] Virendra. Vishwakarma, Sujata Pandey and M. N. Gupta," An Illumination Invariant Accurate Face Recognition with Down Scaling of DCT Coefficients", Journal of Computing and Information Technology - CIT 18, 2010, 1, 53-67

[19] Weilong Chen, Meng Joo Er, and Shiqian Wu," Illumination Compensation and Normalization for Robust Face Recognition Using Discrete Cosine Transform in Logarithm Domain", IEEE TRANSACTIONS ON SYSTEMS, MAN, AND CYBERNETICS—PART B: CYBERNETICS, VOL. 36, NO. 2, APRIL 2006 
[20] K. Delac, M. Grgic, T. Kos, "Sub-Image Homomorphic Filtering Technique for Improving Facial Identification under Difficult Illumination Conditions", International Conference on Systems, Signals and Image Processing (IWSSIP'06), September 21-23, 2006. Budapest, Hungary

[21] YALE FACE DATABASE B, http ://cvc.y ale.edu/projects/y alefacesB/y alef acesB.html, 2001.

[22] Shahrin Azuan Nazeer, Nazaruddin Omar And Marzuki Khalid,"Face Recognition System Using Artificial Neural Networks Approach", IEEE - ICSCN 2007, MIT Campus, Anna University, Chennai, India. Feb. 22-24, 2007. Pp.420425 .

Tripti Goel received her degree in Electronics and Communication from HCE, Sonepat, Haryana, India, in 2004; M.E. in Electronics and Communication from CRSCE, Murthal, Hary ana, India in 2008. she is presently working towards her $\mathrm{Ph}$. D. degree from BPS Mahilla Viswavidyalya, Khanpur, Hary ana,
India. Her present research interests include digital signal processing, and face recognition.

Virendra P. Vishwakarma received his B.Tech. degree in Electrical Engineering from H.B.T.I. Kanpur, Uttar Pradesh, India, in 1994; M.E. in Computer Science \& Engineering from M.N.N.I.T Allahabad, Uttar Pradesh, India, in 2002. He has submitted his Ph.D. thesis in School of Engineering and Technology, Guru Gobind Singh Indraprastha University, New Delhi, India. From 1996 to 2000, he worked for Research Design and Standards Organization, Ministry of Railways, India, where he was involved in many research projects. He also worked in Research \& Development division of Himachal Futuristic Communication Limited, Gurgaon, India. Presently he is working as Assistant Professor in Jaypee Institute of Information Technology, Sector 128, Noida, U.P., India. His research interests include digital image processing, pattern recognition (face recognition) and soft computing. $\mathrm{He}$ is member of IACSIT, ITHEA and ACEEE. 